\section{EXPANDING AND CHARACTERIZING TUMOR INFILTRATING LYMPHOCYTES FROM MYXOFIBROSARCOMA AND UNDIFFERENTIATED PLEOMORPHIC SARCOMA}

${ }^{1}$ Jacky Chen*, ${ }^{2}$ Jay Wunder, ${ }^{2}$ Kim Tsoi, ${ }^{1}$ Nalan Gokgoz, ${ }^{1}$ Irene Andrulis. 'Lunenfeld Tanenbaum Research Institute, Toronto, Canada; ${ }^{2}$ Sinai Health System, Toronto, Canada

Background Sarcoma is a group of rare bone and soft tissue tumors with over 50 distinct subtypes. Survival rate ranges widely due to the lack of efficacious treatments. Immunotherapy, such as adoptive cell therapy (ACT), has drawn significant interest due to its minimal toxicity. In ACT, tumor infiltrating lymphocytes (TILs) are isolated from patients, expanded, and autologously infused back. We recently observed TILs' presence in Undifferentiated Pleomorphic Sarcoma (UPS) and Myxofibrosarcoma (MFS) tumors and found that tumor's PDL1 overexpression is correlated with better clinical outcome in UPS but not MFS. ${ }^{1}$ The Th1 anti-tumoral inflammatory pathway was highly activated in the former cohort, which may explain the favorable outcome. We hypothesize that there are phenotypic and functional differences between TILs of UPS with differential PD-L1 expression, which may be related to clinical outcomes. However, sarcoma TILs are rare and challenging to culture, which significantly impedes their studies. We first aim to robustly expand sarcoma TILs to sufficient numbers.

Methods Tumors' PD-L1 expression was determined by RTqPCR (table 1). To initiate the tumor-fragment (TF) method of TIL culturing, primary tumors were fragmented and cultured in IMDM, IL-2, and 10\% HSA. We further optimized the TF protocol to expand rare sarcoma TILs. Rapid expansion protocol (REP) with anti-CD3/anti-CD28 co-stimulating beads was employed for additional expansion. During REP, TILs were co-treated with gamma-chain cytokines (IL-2, 7, 15, 21).
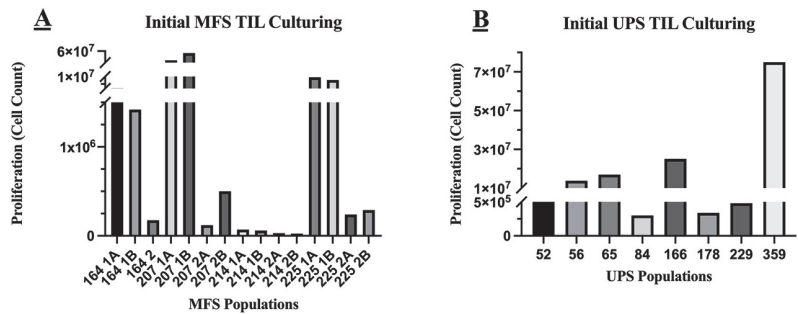

Abstract 168 Figure 1 Initial TIL Culturing with Tumor Fragment Method. Figure A.. The traditional tumor fragment protocol was used to expand TILs of four MFS cases. TILs were cultured and expanded from primary tumor fragments in IL-2 $(6000 \mathrm{IU} / \mathrm{mL})$ supplemented complete media (CM) over four weeks in duration. Fifteen TIL populations were derived from the four MFS cases. Populations were categorized based on their growth rates and labeled as ' 1 ' or ' 2 ' representing 'fast' or 'slow' growing TILs, respectively. Additional populations ' $A$ ' and ' $B$ ' represent biological replicates. Population TIL164 '2' had no replicates. At Week 4, populations' cell counts were determined via hemocytometer. As shown, only 6 out of 15 populations achieved $>1 \times 10^{\wedge} 6$ cells ( $40 \%$ success rate). Figure B. An optimized tumor fragment protocol was used to expand TILs of eight UPS cases. Optimization includes shortening the culturing duration from four weeks to two weeks, reducing frequency of cell culture disruption, and adjusting cell culture environments. TILs were expanded from primary tumor fragments in CM over two weeks in duration. At Week 2, populations' cell counts were determined via hemocytometer. As shown, 5 out of 8 cases achieved $>1 \times 10^{\wedge} 6$ cells ( $62.5 \%$ success rate).

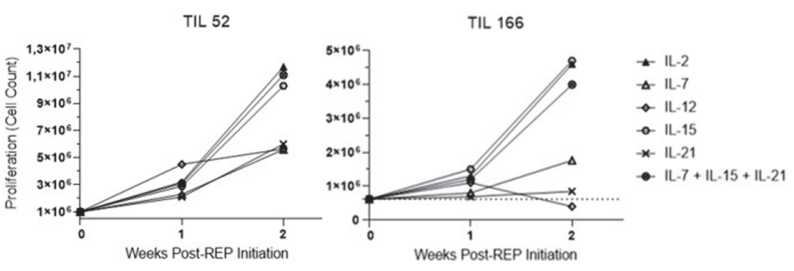

Abstract 168 Figure 2 Gamma-chain cytokine treatments of UPS TILs. Gamma-chain cytokine treatments of UPS TILs with CD3/CD28 stimulation. Magnetic Dynabeads coated with CD3 and CD28 monoclonal antibodies were used to stimulate cells at a bead to cell ratio of 1:3. In ACT, IL-2 is a gold-standard cytokine that facilitates potent T-cell growth. However, it is known to cause activation-induced cell death. Resulting TIL population also possesses an exhaustedeffector phenotype with low durability. UPS TIL Case 52 and Case 166 were treated with various interleukins during two weeks of REP, including gamma-chain IL-7, 15, 21 and inflammatory IL-12. IL-7, IL-12, and IL-21 individually did not elicit significant T-cell growth. IL-15 alone and in combination with IL-7 and IL-21 yield growth comparable to IL2. IL-2 was obtained from Novartis $(50 \mathrm{ng} / \mathrm{mL})$. All other cytokines were obtained from PeproTech $(25 \mathrm{ng} / \mathrm{mL})$.

Abstract 168 Table 1 Tumor PD-L1 RNA Expression. Four MFS and eight UPS cases were processed. Tumors' PD-L1 RNA expression was determined via RT-qPCR and evaluated as a ratio with the housekeeping gene STAM2. TIL359's PD-L1 status has yet to be evaluated.

\begin{tabular}{|c|c|c|c|}
\hline MIF Cases & \multicolumn{1}{c|}{ PD-L1/STAM2 } & UPS Cases & PD-L1/STAM2 \\
\hline TIL 164 & 0.43 & TIL 52 & 0.1 \\
TIL207 & 0.094 & TIL 56 & 0.81 \\
TIL214 & 0.37 & TIL 65 & 0.52 \\
TIL225 & 0.143 & TIL 84 & 0.16 \\
& & TIL 166 & 3.25 \\
& & TIL 178 & 2.49 \\
& & TIL 229 & 0.26 \\
& & TIL 359 & n/a \\
\cline { 1 - 1 } & & &
\end{tabular}

Results Of the 15 MFS TIL populations expanded, only 40\% achieved sufficient growth $\left(1 \times 10^{6}\right)$ for analysis (figure $\left.1 \mathrm{~A}\right)$. Our optimized TF protocol expanded TILs from 8 UPS cases with a $62.5 \%$ success rate (figure 1B). UPS TILs were further stimulated with REP and various gamma-chain cytokine treatments. In ACT, prolonged culturing with IL-2 is known to cause activation-induced cell death, problematic in clinical treatments. We demonstrated that treatments with a Trio-cocktail (IL-7, 15, and 21) or IL-15 alone can achieve TIL proliferation comparable to that of IL-2 (figure 2).

Conclusions Sarcoma infiltrates are difficult to culture, and their roles remain largely unstudied. By optimizing the TF protocol in conjunction with anti-CD3/CD28 treatments, we developed a robust in vitro pipeline to expand rare sarcoma TILs, enabling downstream characterization. We also demonstrated the potential for alternate gamma-chain cytokines to favorably replace IL-2 during TIL expansion. Future phenotypic and functional evaluation of UPS TILs would elucidate the impact of tumors' differential PD-L1 expression on UPS patients' prognoses. These findings would inform the implementation of ACT in sarcoma treatments. 


\section{REFERENCES}

1. Wunder J, Lee M, Nam J, Lau B, Dickson B, Pinnaduwage D, Bull S, Ferguson P, Seto A, Gokgoz N, Andrulis I. Osteosarcoma and soft-tissue sarcomas with an immune infiltrate express PD-L1: relation to clinical outcome and Th1 pathway activation. Onco Immunology 2020;9: e1737385-1- e1737385-13.

Ethics Approval Patients provided signed consent before study entry, as approved by Mount Sinai Hospital's Ethics Board (REB\#01-0138-U).

http://dx.doi.org/10.1136/jitc-2021-SITC2021.168 